\title{
An Initial Investigation into Attribution in SCADA Systems
}

\author{
Andrew Nicholson \\ Cyber Security Centre \\ De Montfort University \\ UK \\ abn@dmu.ac.uk
}

\author{
Helge Janicke \\ Software Technology Research Laboratory \\ De Montfort University \\ UK \\ heljanic@dmu.ac.uk
}

\author{
Tim Watson \\ Cyber Security Centre \\ De Montfort University \\ UK \\ tw@dmu.ac.uk
}

\begin{abstract}
Supervisory control and Data Acquisition (SCADA) systems play a core role in a nation's critical infrastructure, overseeing the monitoring and control of systems in electricity, gas supply, logistics services, banks and hospitals. SCADA systems were once separated from other networks and used proprietary communications protocols, hardware and software. Nowadays modern SCADA systems are increasingly directly or indirectly connected to the Internet, use standardised protocols and commercial-off-the-shelf hardware and software. Attacks on these systems have the potential for devastating consequences and attribution of attacks against SCADA systems presents new challenges. This paper investigates the use of techniques to attribute cyber attacks against SCADA systems. We investigate the use of five known technical attribution techniques in SCADA systems.
\end{abstract}

SCADA, Critical Infrastructure, Attribution, Cyber, Attacks

\section{INTRODUCTION}

SCADA systems are responsible for the monitoring and control of a wide range of a nation's critical infrastructure, including electricity, gas supply, logistics services, banks and hospitals. As such, successful attacks against these systems could have a devastating effect. In the past these systems were separated from other networks, proprietary communication protocols, hardware and software were used, and attacks were of a physical nature. Nowadays there is significant evidence showing that these systems are directly or indirectly connected to the Internet (Radvanovsky and Brodsky 2013). This evidence also shows that these systems are protected with weak or no authentication. ICS-CERT, the U.S. computer emergency response team for industrial control systems, reported that attacks against critical infrastructure are increasing each year (ICS-CERT 2011). 2011 saw a reported 198 incidents, as shown in Figure 1.

Commercial-off-the-shelf SCADA hardware and software are used to cut costs and reduce time to market. This lowers the skill level that is required of an adversary to target such systems and means that anybody with the money and motivation can purchase the hardware and software that is used throughout the critical infrastructure.

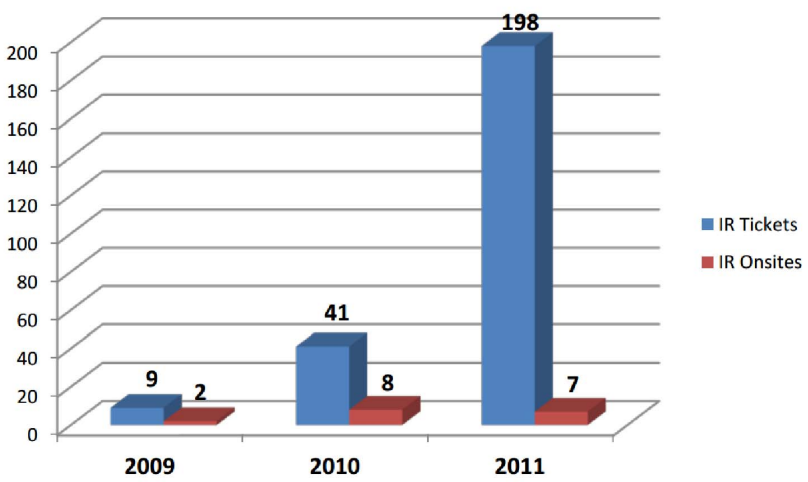

Figure 1: Increase in reported SCADA incidents (ICSCERT 2011)

Attacks against these systems are becoming so common that they are now included in point-andclick penetration testing tools, such as Metasploit, significantly reducing the level of expertise required.

Protecting the systems that monitor and control critical infrastructure has become a primary concern for lawmakers and politicians. The EU Cyber Security Strategy states that critical infrastructure incidents must be reported to a competent authority, while in the U.S., President Obama has signed 
an order which promotes information sharing on incidents (Euractiv 2013).

Identifying the perpetrator of a cyber attack, known as attribution, is a difficult and ongoing problem in the traditional IT domain. Adversaries are able to route attacks through a maze of routers, switches, anonymising services, proxies and compromised hosts, such as with botnets. Adversaries can create malware that morphs or deletes evidence of its presence on a target system. Primary motivators for attribution are (Hunker et al. 2008):

- The prospect of an attacker being identified can serve as a deterrent to future attacks.

- Knowing the identity of an attacker, and information gained in the process of attribution, can be used to improve defensive techniques.

- Attribution, even partial attribution, can provide the basis for interrupting attacks in progress.

Attribution is of significant importance when concerning cyber attacks against SCADA systems and critical infrastructure. Threat actors that are likely to target SCADA systems include nation states, activists, organised criminals, insiders and terrorist groups (Nicholson et al. 2012). Attacks against these systems have the potential to cause physical damage to expensive equipment, wide scale panic and loss of human life. Identifying the perpetrators of attacks or attempted attacks is critical.

This paper investigates the use of techniques to attribute cyber attacks against SCADA systems. Five known attribution techniques are investigated: traceback, deception, digital forensics, network forensics and malware analysis. We limit discussion to a subset of fieldbus protocols and SCADA equipment, selected because of their pervasive use across the critical infrastructure sectors. Despite these restrictions these findings should be applicable at a wider scale. The novelty of this paper is the investigation of attribution techniques from the traditional IT domain applied to the SCADA environment. It highlights that the research and practical work done in this area so far has, almost without exception, been a transfer of traditional IT to SCADA systems and that new techniques, specific to SCADA, should be investigated, while improvements need to be made to existing techniques.

The rest of this paper is structured as follows: Section 2 describes SCADA systems architecture and protocols, Section 3 describes the selection criteria and investigates attribution, Section 4 details related work and Section 5 contains conclusions with our recommendations for SCADA attribution and future work.

\section{SCADA SYSTEMS}

\subsection{History}

Supervisory control systems have been in use since the 1940s, when they consisted of simple relay/tone and pilot wire systems. The first Supervisory Control and Data Acquisition (SCADA) systems emerged in the 1960s, and were composed of communication between a computer master station and many remote terminal units (RTUs), sometimes hundreds. RTUs and their communication protocols were proprietary; specific to the vendor of the master station. Security was considered but only with respect to checksums for ensuring message integrity. Network links were composed of point-topoint wiring using telephone lines and later radio technology for communication between the master station and remote sites. In the 1990s and onwards SCADA systems moved towards standardised protocols and platforms. SCADA systems are now available to buy off the shelf in an open market. While this saves on purchasing and operating costs, it also means that the systems are free to be inspected and probed by any motivated and financed entity. SCADA systems have been directly or indirectly connected to the Internet, driven by the requirement for engineers to perform remote maintenance, rather than travel to remote sites. Researchers have identified that these systems are protected with weak or no authentication (Radvanovsky and Brodsky 2013). Security solutions for insecure legacy SCADA systems have been non-intrusively retrofitted, to prevent disruption to operations.

\subsection{Systems Architecture}

Modern SCADA architectures are composed of three segments: the corporate network segment, SCADA network segment and field devices segment (Nicholson et al. 2012).

Corporate network segment - mostly operate in the same way as a general IT network found in a business. It performs the same day-today business operations such as marketing, email communication, accounting and administrative tasks, hence requiring an Internet connection. Also similar is that these networks may connect to third party cloud-based services such as file or web server providers. This means that the corporate network segment has the same attack surface as a general IT network, such as web application SQL injection, e-mail spear-phishing and exploitation of other vulnerabilities.

SCADA network segment - contains the SCADA system, comprised of servers and workstations that are used by operators to interact with the field 
devices segment. Human Machine Interfaces (HMI) are used by operators to interpret and control PLCs and RTUs. Operators use a software-based graphical user interface that monitors and modifies values, such as motor rotation speed. Validation in software may prevent operators overriding values that could cause harm to machinery or human life. A Historian system collects and stores an audit trail of operational data in a database. Historians may collect information from thousands of devices and therefore need to be able to process large volumes of data in real-time. Backup control centres and data backup centres may also be present. It is at this point and beyond that similarities between general IT networks and industrial networks cease.

Field devices - comprised of Programmable Logic Controllers (PLCs), Remote Terminal Units (RTUs) and Intelligent Electronic Devices (IEDs). IEDs are microprocessor devices, such as sensors, motors, circuit breakers or console lights. These devices are controlled by RTUs and PLCs, primarily with fieldbus protocols. RTUs monitor IEDs and transmit data to PLCs or the SCADA network using protocols such as Modbus and DNP3. PLCs are computers which are able to automate functions primarily using simple ladder logic statements. Sensor data flows from the sensors in the field to the RTUs and PLCs and/or to a data collection point in the SCADA network, where it can be interpreted by an operator using an HMI. The RTUs and PLCs can control parts of the infrastructure directly, such as regulating valves or activating switches through the IEDs, based on either data from the field sensors, or from operator input from the control centre. The resulting control action then flows from the operator or RTU to the IED to make the change to the system. These systems are physically connected by Ethernet, fibreoptic cabling, telephone lines, microwave, satellite or radio. SCADA systems today use standard network topologies such as bus, hub, ring and star and are therefore prone to attacks that rely on these specific topologies to be present.

Figure 2 shows a typical SCADA environment ( $\mathrm{Pa}-$ cific Northwest National Laboratory 2006) and presents the segments, machines and devices that have been discussed.

\subsection{Protocols}

In the corporate network segment the protocols used are essentially the same as in any other corporate network segment e.g. SMTP and IMAP for e-mail, SSH and FTP for file transfer, HTTP for web services, DNS, TCP, UDP and ARP for network operations, primarily within IPv4, although in some cases, IPv6. In the SCADA network and field device segments the protocols are mostly only found within industrial



Figure 2: Typical SCADA System Architecture (Pacific Northwest National Laboratory 2006)

networks e.g. Modbus, DNP3 and ICCP. However, there is some overlap, in some cases PLCs may be accessed by HTTP/HTTPS, SSH, FTP etc. In the past SCADA systems were composed of proprietary protocols. This added a layer of security by obscurity. However, it meant buyers were locked in to one particular vendor. If that vendor were to go bankrupt then support for the technology would be gone. Nowadays open and standardised protocols and architectures are prevalent, meaning that buyers can mix and match equipment and do not suffer from vendor lock-in. It also means that protocols can be scrutinised by the SCADA community and are able to mature. However, researchers highlight that using common, general IT network protocols and software (e.g. Windows NT) increases the chance of an attack (Igure et al. 2006). This stands to reason since a much wider pool of adversaries understand the weaknesses inherent in corporate network protocols and systems, as opposed to those only used in SCADA. With a general understanding of the SCADA history, architecture and protocols, we move on to discuss attribution.

\section{ATTRIBUTION}

\subsection{Overview}

Attribution is used in traditional IT domains to identify the who, what, where, why and how of cyber attacks. It is defined as 'determining the identity or location of an attacker or an attacker's intermediary' (Wheeler and Larsen 2003). Attribution might result in a conclusive answer such as who did it: an individual, a group, a nation state, etc. Attribution may only provide an inconclusive hint and other intelligence/evidence may be required. Successful attribution may identify perpetrators so that they may face prosecution i.e. cyber crime, or be used to plan retaliation, i.e. cyber warfare. Attribution 
results may be brought to public light to embarrass, shame or demonstrate superiority over an adversary.

Attribution takes place after an intrusion or compromise has been identified. In traditional IT environments intrusions are detected using a variety of technical tools such as signature or anomaly-based intrusion detection systems, anti-virus, baselining tools and security information and event management tools. Researchers have modified these tools to detect intrusions in the SCADA environment, however, they must be engineered to parse the unique and sometimes proprietary protocols that are prevalent in the SCADA and fieldbus segments (Verba and Milvich 2008) (Fovino et al. 2010).

A number of traditional security paradigms do not easily transfer to SCADA environments. For example, penetration testing, which involves finding and exploiting vulnerabilities to evaluate security, is an often noisy and disruptive task. SCADA systems are fragile; availability and uptime is of critical importance. SCADA-specific traditional computer security defences, such as firewalls and intrusion detection systems are available. However there is currently little research that considers the feasibility of deploying computer security attribution techniques in these environments.

\subsection{Selection of Attribution Techniques for Investigation}

It is said that 'there are many types of attribution, and different types of attribution are useful in different contexts' (Clark and Landau 2011). We consider a subset of available technical attribution techniques and a taxonomy by Wheeler and Larsen (2003), shown in Figure 3 , presents a useful starting point for this selection. From the possible techniques, five were selected, based on their pervasiveness, diversity and academic interest. They are: traceback, deception, digital forensics, network forensics and malware analysis. In the following subsections the discussion involves the following themes:

- Overview of technology in its traditional domain

- Relevance in the SCADA environment

- Suitability in the SCADA environment based on above points

\subsection{Traceback}

Traceback is a network-based approach to attribution that has received significant interest from the academic community. In almost all proposals this technique involves attributing a source IP address (or closest router) and intermediate router IP addresses to mark the tracks of an attack over the Internet.

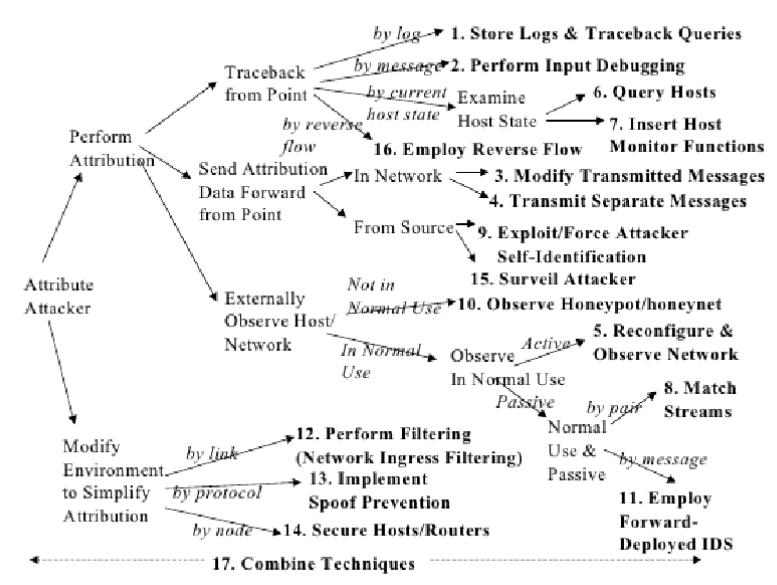

Figure 3: Attribution Taxonomy (Wheeler and Larsen 2003)

The two main traceback proposals are packet logging and packet marking. Packet marking involves marking packets with identifiable information when passing through networking devices, such as routers or switches (Belenky and Ansari 2003). Packets are marked with information that describes the path that the packet has taken. A victim may collect and inspect received packets in order to deduce the attack path. In packet logging routers store information about the packets that have passed through them (Gong and Sarac 2005). During an attack or post-attack, network traffic data stored at each router may be questioned to see if the router was part of the attack path. Traceback in traditional IT domains was primarily proposed to stop ongoing Denial of Service attacks. This type of attack is generally seen as an inconvenience in the traditional domain, however against SCADA systems, could have devastating effects. Therefore it is vital to investigate how this technique could be applied to SCADA networks and protocols.

Packet marking proposals manipulate the content of protocol headers against the spirit of the standards and request for comments (RFCs). Infrequently used packet header fields, such as the IPv4 Identification field, are loaded with traceback data, which in this example removes packet fragmentation functionality. Logistically this is possible with an IPv4 header, but some of the popular fieldbus protocols have limited available space in the packet header. IPv4 and DNP3 packet headers are shown in Figure 4.

Specifications have been defined for a number of SCADA protocols, such as DNP3 and Modbus, to be encapsulated TCP/IP, meaning that packet header space may not be such an issue, providing that this flavour of the protocol is used. 
example, the action event on the HTML forms reads 'action=honeyd-feedback.py', a clear indicator that the SCADA system is actually a honeypot.

DigitalBond (2007) expanded upon the work of Franz and Pothamsetty when they released a SCADA honeypot as a VMWare virtual image. This tool comprised of a SCADA honeypot based on Franz and Pothamsetty's honeypot and a Honeywall to collect data and stop outbound connections from compromised honeypots. The Honeywall could be placed in front of either the SCADA honeypot or a real PLC that is not used for production. This second option was important because it enabled a real SCADA system to be used as a honeypot. Further to this work, Rrushi and Campbell (2008) proposed 'mirage theory' for industrial control systems, in which active decisions are made to draw the adversary towards a honeypot which also used real SCADA physical systems as honeypots. Later in 2012, a different kind of SCADA honeypot was released, which did not focus on the SCADA network protocols. Instead, it focused on a growing threat to SCADA systems; infection by removable media (GhostUSB 2013). Ghost USB Honeypot emulates a USB storage device and captures attempts at infection.

Research on SCADA honeypots so far has shown initial promise, however the majority of proposals have taken known honeypots and tried to fit them into a SCADA environment. Security experts who wish to develop SCADA honeypots should work closely with SCADA engineers in order to create realistic honeypots that can be used effectively.

\subsection{Digital Forensics}

Digital Forensics is a broad subject which involves the recovery, acquisition and investigation of digital evidence. In traditional IT domains commercial tools such as EnCase and FTK and open source tools such as Sleuthkit and Autopsy are used to acquire, analyse, and report on digital evidence. These tools tend to be specific to $x 86$ and $x 64$ processor architectures and targeted towards file systems, such as FAT, NTFS and popular operating systems, such as Windows and Linux.

Forensics in a SCADA environment could identify attribution data to identify perpetrators. In the SCADA network segment and field device segments there are a broad range of devices which may store a wealth of digital evidence. However, SCADA systems come with a unique set of challenges for forensic analysis. For example, the standard forensic procedure for taking a bit-for-bit disk acquisition involves switching off a system, connecting the hard disk to a write blocker and acquisition system and then waiting for the acquisition to complete. Switching off a SCADA system which monitors and controls critical infrastructure is unlikely to be an option. One way to mitigate this issue is to have fail over systems. However, this is costly and if the fail over system is a duplicate of the original system, it might be infected in exactly the same way.

The diversity of devices that a forensic investigator can encounter in the SCADA environment is far wider than that of the traditional IT domain. Traditional IT systems have a lifespan of a couple of years, perhaps 10 at most, while some SCADA systems from the 1960's are still operating. However, as PLCs and other SCADA devices continue to move towards commercialoff-the-shelf hardware and software, the forensic analysis of SCADA systems becomes standardised and therefore simpler.

Among the diverse devices found in SCADA environments is the Historian, which is essentially a database management system (DBMS). It collects a wealth of data to enable auditing, trend analysis and anomaly detection. As a DBMS, traditional database forensics techniques should be suitable for these devices. However, unlike the historian, many of these devices encountered are unlikely to have persistent memory. It is true that 'most process control systems were not built to track their processes, but merely to control them' (Nance et al. 2009). For example, the Siemens $57-300$, shown in Figure 5 , is a popular PLC. For storage, it uses a micro memory card (MMC) which ranges from $64 \mathrm{~KB}$ to $8 \mathrm{MB}$, while integrated CPU memory for this device ranges from $32 \mathrm{~KB}$ to $2 \mathrm{MB}$.



Figure 5: Siemens S7-300 PLC

With this absence of persistent memory, researchers have proposed the use of another technique from the traditional IT domain to SCADA; live forensics. In live forensics data acquisition takes place while the system is operational. In traditional IT systems, tools are used to capture running processes, RAM memory, browsing history and more, in the order of volatility. Performing live forensics on 
an operational machine in a SCADA environment prompts significant challenges; accidentally causing the machine to crash could be catastrophic. Ahmed et al. (2012) discusses this issue and suggests using fail over systems to allow for live forensic analysis to take place. Another challenge is that post-incident the investigator is competing with recovery efforts which will most likely destroy evidence. There is also clearly a logistics concern when performing SCADA forensics. Field devices could be located many miles away, perhaps on different continents, or perhaps in difficult to reach places, such as on the ocean floor. Physically reaching these devices may not be possible.

Forensics is primarily a practitioner-led field with research taking place as and when it is required. In a recent effort to outline a research agenda for this field, SCADA forensics was identified as a predominant theme (Nance et al. 2009). The following points were identified as near future research for forensics in SCADA systems:

- Collection of evidence in the absence of persistent memory

- Hardware-based capture devices for control systems network audit trails

- Honeypots for control systems as part of the investigatory process

- Radio frequency forensics

- Intrusion detection systems for control systems

\subsection{Network Forensics}

Another field of forensics used in traditional IT systems is network forensics. This field primarily involves two stages: collecting network messages and analysing network messages. Existing infrastructure such as switches and routers can be configured to collect messages, or extra equipment can be deployed, such as a network tap device. By logging messages to files, analysis can take place during an attack or post-attack. During analysis of network traffic, attribution data can be found, such as connection source, time of connection, commands that were sent and payload data.

Collection of data is relatively straightforward. An organisation must identify points in the network were they wish to collect network data. Mahmood et al. (2010) describes traditional network analysis problems and network sniffer deployment in a SCADA environment. An area that will require further consideration is when traditional communication channels other than Ethernet are used, such as RS232 and radio link. Specialist sniffers will be required in this instance. Traffic should be stored in known network capture formats, such as PCAP. Wireshark, a popular network sniffer and packet analyser tool, already has dissectors for some SCADA protocols, including Modbus, DNP3 and FINS, a proprietary protocol, however there are many SCADA protocols that are not supported.

Full packet capture in a traditional IT system can cause problems due to the high volume and large packet size. In a SCADA environment traffic volume is generally much lower and message sizes are much smaller. Message content is likely to be significantly less diverse, as content is machine generated and not user generated. This results in network collection devices requiring less storage and processing power, meaning that organisations can make savings or deploy more devices. Passive devices with adequate throughput should be used for full packet capture so that performance is not affected and extra traffic is not generated; both are critical points in the often fragile SCADA and fieldbus network segments. Retrofitting of these devices into existing and operational SCADA environments should be done in a non-intrusive manner to prevent disruptions to ongoing operations.

Of course, similar to traceback, network forensics will only be able to identify attacks that use network communications as a vehicle for attack. Those that use removable media will not be visible.

\subsection{Malware Analysis}

Malware, in it's various forms; virus, worm, trojan, adware, spyware, back doors and rootkits, may be analysed to identify characteristics which could be used as an attribution data source. Malware analysis in the traditional IT domain can be split into two areas: behavioural analysis and code analysis.

Behavioural analysis examines the way that malware interacts with the environment. Malware might make changes to the registry, create new processes, hide files, execute other binaries, contact command-andcontrol servers, cover tracks by deleting evidence of its modifications (as Stuxnet did), disable security protections, record user interaction (e.g. keylogging), harvest sensitive data, exfiltrate data, attempt to update, pivot to other systems, establish back doors and more. A controlled sandbox environment is usually created to examine this behaviour. Virtual machines are commonly used for this task as they can be quickly reset with snapshot/roll-back functionality. A wide range of tools are available to analyse malware behaviour in the traditional IT domain, such as the Windows SysInternals suite. The investigator can change the sandbox environment to illicit a response from malware. Examples of change include: 
- Introducing new services, files and removable media

- Introducing Internet connectivity

- Browsing websites, sending and receiving e-mail

- Inputting passwords and other sensitive information

The response or lack of response helps to identify what the malware does. The process of behavioural analysis can be automated with tools such as CWSandbox which monitors Windows system calls made by malware. Behaviour analysis tools and environments are fairly limited to operating systems used in traditional IT environments e.g. Windows and Linux; they do not support the firmware found on SCADA PLCs and RTUs. Ahmed et al. (2012) identified that SCADA simulation environments should be created, possibly by Universities and industry partners, and this would certainly help to rectify this issue.

Code analysis is concerned with examining the code that makes up the malware. Source code for malware might be available, although it is unlikely. If by chance it is then source code analysis can take place. Otherwise, reverse engineering and debugging take place. Reverse engineering involves restoring the malware's binary machine code to human-readable assembly code, using tools such as IDA Pro and OllyDbg. These tools are particularly effective at reversing binaries compiled for $x 86$, x64 and ARM CPU architectures. The code can then be executed in a debugger to step through the instructions, inspect register contents, identify embedded strings and set breakpoints to figure out exactly what the malware does.

Practitioners used reverse engineering against the Stuxnet malware (Falliere et al. 2011). They identified clues in the code, such as binary compile times, suspicious variable names, registry keys that appear to be dates and directory names that might be biblical names. Some or all of these clues could have been false flags; data that was purposely crafted to implicate another entity as the malware authors. Symantec consulted the expertise of SCADA practitioners, such as Ralph Langner, in order to understand the effects that the Stuxnet malware had on the Siemens PLCs. This again highlights the diverse skill sets required for the SCADA environment and the necessity for security professionals to work closely with SCADA engineers. Code analysis was also used to identify re-use of code and libraries; Stuxnet, Flame and Duqu were identified as having shared code.

\section{RELATED WORK}

SCADA security has experienced a flux of interest, particularly following the widely publicised Stuxnet malware. Attribution of SCADA attacks has been pioneered by practitioners involved in the analysis of Stuxnet and other recent widely publicised malware (Falliere et al. 2011). Attribution of these attacks has been accompanied by non-technical means such as 'insider accounts' (Sanger 2012). There has been significant research interest in the use of traditional IT security paradigms, such as firewalls and intrusion detection systems, deployed in SCADA environments. While with respect to attribution, Hunker et al. (2009) discussed techniques, legal issues and proposed a voluntary network of attributable activity.

\section{CONCLUSION}

In this paper we have investigated the use of a subset of attribution techniques that are used in the traditional IT domain, when used in the SCADA domain. There are many more attribution techniques that could form further investigation e.g. Wheeler and Larsen (2003). Technical controls for performing attribution are just one aspect of a wider solution. Non-technical measures, such as cuo bono analysis and following the money are used to attribute attacks. Asking questions such as 'Who has the resources?' and 'Who has the motive?' serve to logically identify perpetrators.

Attribution should not be seen as a single process or problem, but rather a jigsaw puzzle in which there are many attribution pieces. Different techniques, both technical and non-technical, are able to yield different pieces of the puzzle. While it is unlikely that all of the pieces will be collected following an intrusion, more pieces help to provide a better overall picture. A theory of deduction, then, is helpful to logically understand this attribution puzzle and further research into attribution techniques that do and do not work well together would be a useful exercise.

As the number of SCADA incidents continues to increase as shown in ICS-CERT (2011), the ability for nations to attribute attacks against their critical infrastructure becomes evermore important. By reviewing the selected subset of attribution techniques it can be seen that there are a few techniques that have been modified for use on SCADA systems but that tools and techniques specifically designed for them are lacking. It is equally clear that there is an urgent need for an effective set of SCADA attribution tools. This paper has highlighted the challenges associated 
with transferring techniques designed for traditional IT systems and the Internet to SCADA systems. An outcome of this work is a clear requirement for more techniques to be developed for, or modified for use on, SCADA systems. Similarly there is a juxtaposition of these two domains, where traditional IT domains and SCADA domains connect. Attribution solutions need to account for malware like Stuxnet, which is able to transcend both of these domains, exploiting vulnerabilities in the corporate network segment and the SCADA and fieldbus segments. Effective solutions will ensure that security experts and SCADA engineers collaborate closely. For example, a honeypot design that has significant input from both parties is likely to appear far more realistic to an adversary. A workshop to identify a forensics research agenda took place (Nance et al. 2009). A similar workshop for attribution and SCADA systems would be a productive exercise, providing that the right balance of individuals were present, for example, technical security specialists, SCADA experts and legal experts.

\section{REFERENCES}

Irfan Ahmed, Sebastian Obermeier, Martin Naedele, and Golden Richard III. SCADA systems: Challenges for forensic investigators. 2012. ISSN 0018-9162.

Andrey Belenky and Nirwan Ansari. IP traceback with deterministic packet marking. Communications Letters, IEEE, 7(4):162-164, 2003. ISSN 1089-7798.

Steven Michael Bellovin, Marcus Leech, and Tom Taylor. ICMP traceback messages. 2003.

David D Clark and Susan Landau. Untangling attribution. Harv. Nat'l Sec. J., 2:323, 2011.

DigitalBond. SCADA honeynet, 2007. URL http://www . digitalbond. com/tools/scadahoneynet/. Accessed 02.05.2013.

Dionaea. Honeypot. Website, 2013. URL http://dionaea.carnivore.it. Accessed 02.05.2013.

Euractiv. EU, US go separate ways on cybersecurity, $2013 . \quad$ URL http://www . euractiv. com/specialreport-cybe rsecurity/eu-us-set-different-approach-cyb -news-518252. Accessed on 02.03.13.

Nicolas Falliere, Liam O Murchu, and Eric Chien. W32. stuxnet dossier. White paper, Symantec Corp., Security Response, 2011.
Igor Nai Fovino, Andrea Carcano, $\mathrm{T}$ De Lacheze Murel, Alberto Trombetta, and Marcelo Masera. Modbus/DNP3 statebased intrusion detection system. In Advanced Information Networking and Applications (AINA), 2010 24th IEEE International Conference on, pages 729-736. IEEE, 2010.

GhostUSB. Honeypot. Website, 2013. URL http://code.google.com/p/ghost-usb-honeypot. Accessed 02.05.2013.

Chao Gong and Kamil Sarac. IP traceback based on packet marking and logging. In Communications, 2005. ICC 2005. 2005 IEEE International Conference on, volume 2, pages 1043-1047. IEEE, 2005.

Honeyd. Honeypot. Website, 2013. URL http://www . honeyd.org/. Accessed: 02.03.13.

Jeffrey Hunker, Bob Hutchinson, and Jonathan Margulies. Role and challenges for sufficient cyber-attack attribution. Institute for Information Infrastructure Protection, 2008.

Jeffrey Hunker, Robert Hutchinson, and Jonathan Margulies. Attribution of cyber attacks on process control systems. In Critical Infrastructure Protection II, pages 87-99. Springer, 2009.

ICS-CERT. Incident response summary report. Technical report, U.S. Department of Homeland Security, 2011.

Vinay M Igure, Sean A Laughter, and Ronald D Williams. Security issues in SCADA networks. Computers \& Security, 25(7):498-506, 2006. ISSN 0167-4048.

Abdun Naser Mahmood, Christopher Leckie, Jiankun $\mathrm{Hu}$, Zahir Tari, and Mohammed Atiquzzaman. Network traffic analysis and SCADA security. In Handbook of Information and Communication Security, pages 383-405. Springer, 2010.

Kara Nance, Brian Hay, and Matt Bishop. Digital forensics: defining a research agenda. In System Sciences, 2009. HICSS'09. 42nd Hawaii International Conference on, pages 1-6. IEEE, 2009.

Andrew Nicholson, Stuart Webber, Shaun Dyer, Tanuja Patel, and Helge Janicke. SCADA security in the light of cyber-warfare. Computers and Security, 31(4):418-436, 2012. ISSN 0167-4048.

U. D. o. E. Pacific Northwest National Laboratory. The role of authenticated communications for electric power distribution, 2006. URL http://www. truststc. org/scada/papers/paper 34.pdf. 
Venkat Pothamsetty and Matthew Franz. SCADA honeynet project: Building honeypots for industrial networks, 2004.

Bob Radvanovsky and Jake Brodsky. Informationweek.com, January 2013. URL http:// www.informationweek.co.uk/government/ security/thousands-of-industrial-control -systems/240146091. Accessed 02.05.2013.

J Rrushi and Roy Campbell. Using deception to facilitate intrusion detection in nuclear power plants. In Proceedings of the Third International Conference on Information Warfare and Security, 2008.
David E Sanger. Confront and Conceal: Obama's Secret Wars and Surprising Use of American Power. Random House Digital, Inc., 2012.

Lance Spitzner. Honeypots: Catching the insider threat. In Computer Security Applications Conference, 2003. Proceedings. 19th Annual, pages 170-179. IEEE, 2003.

Jared Verba and Michael Milvich. Idaho national laboratory supervisory control and data acquisition intrusion detection system (SCADA IDS). In Technologies for Homeland Security, 2008 IEEE Conference on, pages 469-473. IEEE, 2008.

David A Wheeler and Gregory N Larsen. Techniques for cyber attack attribution. Technical report, 2003. 